\title{
Informatics and Integrated Computational Materials Engineering: Part II
}

\section{Krishna Rajan}

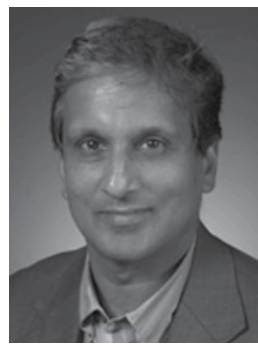

This issue of $J O M$ presents the second in a twopart series on materials informatics. In the previous issue (March 2008), some of the basic concepts of what constitutes informatics were introduced. In this issue, we continue that theme with papers that serve to emphasize the diversity of topics that encompass the field of informatics. The timing of the publication of these $J O M$ topics on materials informatics coincides with the issuing of a report of the National Materials Advisory Board on Integrated Computational Materials Engineering (ICME). ${ }^{1}$ This report defines that information as including "curated data sets, structure-property models, processing-structure relationships, physical properties and thermodynamic, kinetic and structural information." It provides a good summary of the needs, status, and challenges the materials engineering community faces to generate this information. The report defines ICME as "the integration of materials information, captured in computational tools, with engineering product performance analysis and manufacturing-process simulation." The report envisions an ICME system as one that integrates many different components of information that are "linked by means of a software integration tool to a designer knowledge base containing tools and models from other engineering disciplines." At the core of this integration the report identifies what it calls "use cases" which serve as the conduit for information from databases, models, and computational tools to achieve a knowledge base that can solve materials engineering problems.

So where does informatics fit into this framework? I would suggest that the articles in this and the previous companion JOM issue serve to emphasize that informatics can in fact be that "hub" (Figure 1). Each paper in these issues links one or more of the compo-

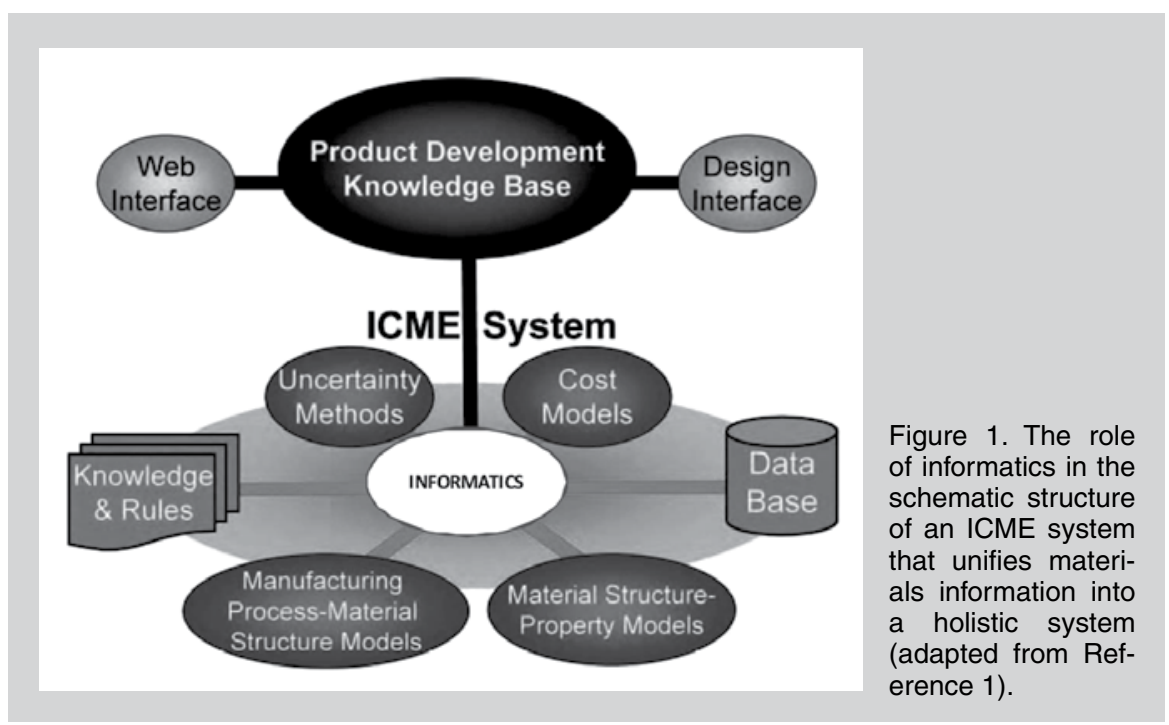

nents identified in the ICME report.

In this issue, C. Suh and K. Rajan explore ways to discover knowledge and rules associated with crystal chemistry strictly by interrogating databases using data-dimensionality-reduction techniques and discover new structurechemistry relationships. Hence materials informatics plays a key role for ICME, namely, by providing the tools to achieve that integration.

Having ways to generate information is usually viewed as an important goal but B. Ganapathysubramanian addresses a critical and very practical issue: we may not always be able to collect all the information we need. He shows how one can use stochastic modeling to deal with uncertainty caused by missing information. This then permits one to develop or enhance and refine materials-based models.

R. Hrubiak and colleagues challenge the conventional notion of databases as simple repositories of information. They provide a good example of how to organize a database so that it can be converted to a "knowledge base." When providing the right type of web interface, they show how complex and integrated information can be made easily accessible.

\section{Reference}

1. Committee on Integrated Computational Materials Engineering, National Research Council, Integrated Computational Materials Engineering: A Transformational Discipline for Improved Competitiveness and National Security (Arlington, VA: National Academy Press, 2008).

Krishna Rajan is with the Department of Materials Science and Engineering, lowa State University, 2220 S. Hoover Hall, Ames, IA 50011; e-mail krajan@iastate.edu. He is advisor to JOM from the Computational Materials Science and Engineering Committee. 